\title{
Effects of whey protein supplement in the elderly submitted to resistance training: systematic review and Meta-analysis.
}

\author{
Tamy Colonetti ${ }^{1 \dagger *}$, Antônio Grande ${ }^{2 \dagger}$, Karen Milton ${ }^{3 \dagger}$, Charlie Foster $^{4 \dagger}$, Maria Inês da Rosa ${ }^{5 \dagger}$ \\ ${ }^{1} \mathrm{MSc}$ student, Graduate Program in Health Sciences, Laboratory of Epidemiology, Universidade do Extremo \\ Sul Catarinense, Criciuma, SC, Brazil; tamycolonetti@hotmail.com \\ ${ }^{2} \mathrm{PhD}$, Associate Professor, Laboratory of Evidence-based practice, Universidade do Extremo Sul \\ Catarinense, Criciuma, SC, Brazil; grandeto@gmail.com \\ ${ }^{3} \mathrm{PhD}$, Post-doctoral Fellow, British Heart Foundation Centre on Population Approaches for Non- \\ Communicable Disease Prevention, Nuffield Department of Population Health, University of Oxford. \\ karen.milton@dph.ox.ac.uk \\ ${ }^{4} \mathrm{PhD}$, University Research Lecturer \& Programme Leader, British Heart Foundation Centre on Population \\ Approaches for Non-Communicable Disease Prevention, Nuffield Department of Population Health, \\ University of Oxford; charlie.foster@dph.ox.ac.uk \\ ${ }^{5} \mathrm{MD}, \mathrm{PhD}$, Associate Professor, Graduate Program in Health Sciences, Laboratory of Epidemiology, \\ Universidade do Extremo Sul Catarinense, Criciuma, SC, Brazil; mir@unesc.net
}

* Correspondence: tamycolonetti@hotmail.com; Tel.: +55-048-3431-2741

\begin{abstract}
We performed a systematic review to map the evidence and analyze the effect of whey protein supplementation in the elderly submitted to resistance training. Methods: A comprehensive search on Medline, LILACS, EMBASE and the Cochrane Library for relevant publications was conducted until August 2015. The terms used in the search were: "Resistance Training"; "Whey protein"; "elderly". Two authors independently assessed abstracts, texts in full and made critical appraisal. Results: A total of 632 studies were screened. Five studies were included composing a sample of 391 patients. The supplement whey protein was associated with higher total protein ingestion $9.40(95 \% \mathrm{Cl} 4.03$ to 14.78$)$, and with an average change in plasma leucine concentration ranging from $406 \mu \mathrm{mol} / \mathrm{L}$ to $490 \mu \mathrm{mol} / \mathrm{L}$ compared with the control group. The supplementation was also associated with increased mixed muscle protein synthesis 1.26 (95\% $\mathrm{Cl} 0.46$ to 2.07$)$ compared to control group. Just one trial compared lean body mass in the group receiving whey protein supplementation and the control group $(n=80) 0.07(95 \% \mathrm{Cl}-0.37$ to 0.51$)$. Conclusion: We observed an increase in total protein intake, resulting in increased concentration of leucine and mixed muscle protein synthesis rate. No significant increase related to muscle mass or strength were observed.
\end{abstract}

Keywords: systematic review; whey protein; meta-analyses; elderly. 


\section{Introduction}

It is widely accepted that resistance training promotes increased muscle strength and skeletal muscle mass. In the aging context, resistance training has a special importance after 60 years old, when changes in body composition are accelerated due to the aging process [1]. The aging process is associated with the development of chronic diseases due to biopsychological changes in the body. Among these changes are processes related to changes in body composition such as skeletal muscle mass reduction [2].

In response to resistance training, muscle activation and damage influence protein turnover [3]. If the amino acids in the body have insufficient levels, it results in negative protein balance, leading to harmful side effects such as loss of muscle mass and late period recovery [4]. Whey protein is widely available as a dietary supplement claimed for building muscle mass. Considering the rapid rate of digestion, this supplement provides a quick source of amino acids that can be absorbed by the muscles to repair and rebuild muscle tissue [5]. Protein metabolism during and after exercise is affected by different factors such as: gender; age; physiological changes that interfere in the absorption of nutrients; intensity, duration and type of exercise; and the consumption and availability of energy and carbohydrates daily. As the population is getting older, the evidence from resistance exercise and whey protein needs to be synthesized. Therefore, we performed a systematic review of the literature to map the evidence and analyze the effect of whey protein supplementation in the elderly submitted to resistance training.

\section{Materials and Methods}

\section{Search Strategy}

We performed a systematic review following the PRISMA-statement guidelines [6]. The review protocol is registered at PROSPERO (International prospective register of systemic reviews, http://www.crd.york.ac.uk/prospero; CRD42014014317). We searched the following databases: Medical Literature databases Analysis and Retrieval System Online (Medline) through Pubmed, Latin American and Caribbean Health Sciences (LILACS), Excerpta Medical Database (EMBASE), and the Cochrane Library for relevant publications until August 2015. We searched using the following terms: "Resistance Training", "Whey protein" and "elderly", additionally synonyms were included in the search strategy The search was limited to human studies and had no language restrictions. Reference lists of all primary studies were reviewed to identify additional relevant citations. The complete search strategy is available on request.

\section{Screening of abstracts for eligibility}

Abstracts/titles identified from the search were screened by two reviewers (TC and AJG) using covidence (http://www.covidence.org), an online management tool for systematic reviews. Disagreements about study inclusion or exclusion were resolved by consensus, and when this was not possible, through consultation with a third reviewer (MIR).

\section{Study Selection}

Design - We included only randomized controlled trials of elderly patients treated with whey protein supplementation in combination with resistance training.

Patients - We analyzed studies that included elderly individuals over 60 years.

Intervention- Resistance training compared to a control group. The selected studies must explain in detail dose and time of administration of Whey protein supplementation in the elderly, and also what was the training protocol which was undertaken in order to gain muscle mass. 
Two investigators (TC, AJG) independently extracted data from the primary studies. We extracted data on study, patient's characteristics, and intervention, using a standardised form. Inter-rater agreement was calculated to observe agreement among assessors.

\section{Quality assessment}

All included studies were assessed for their methodological quality. The Cochrane Collaboration's risk of bias tool was used (RevMan 5.3).

\section{Publication Bias}

It was not possible to test for funnel plot asymmetry due to the low number of included studies. According to the Cochrane Handbook for Systematic Reviews of Interventions, it is recommended that meta-analyses include at least 10 studies.

\section{Data Synthesis and Statistical Analysis}

Meta-analysis was possible only for three outcomes: total protein ingestion, leucine, and mixed muscle protein synthesis rate. Outcomes that could not be analyzed by meta-analysis, including lean mass and fat mass, strength, and adverse events, were described in the text. We used mean and standard deviations to derive a standard mean difference (SMD) with 95\% confidence intervals using RevMan software. Pooled-effect estimates were derived using a random-effects model with Mantel-Haenszel statistics. Study heterogeneity was determined using the 12 statistic (in which numbers greater than $75 \%$ suggest considerable heterogeneity) [7] and $P$ values from the $X 2$ test. In cases of considerable heterogeneity, no pooled estimate was calculated.

\section{Results}

The search identified a total of 656 studies; 24 studies were duplicates. A total of 632 studies were screened for titles and abstracts. Of these, 612 were excluded because they were reviews, animal experiments, observational studies, or conducted with an adult population (under 60 years). A total of 20 studies were analyzed in full-text. From these studies, 15 full articles were excluded for not meeting criteria for: study design; population studied; intervention; or outcomes. The study selection process is summarized in Figure 1. 

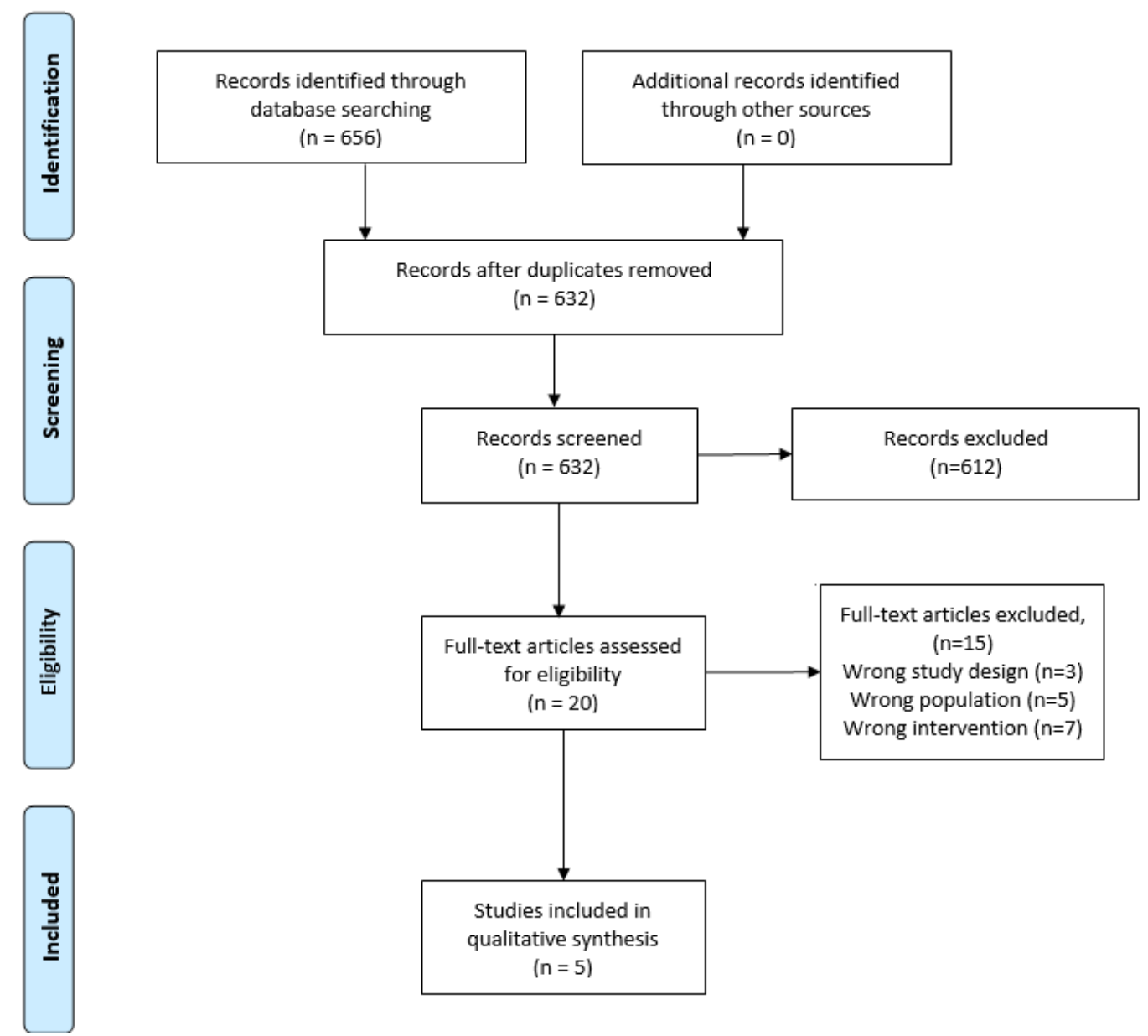

Figure 1. Flow chart of study selection. 


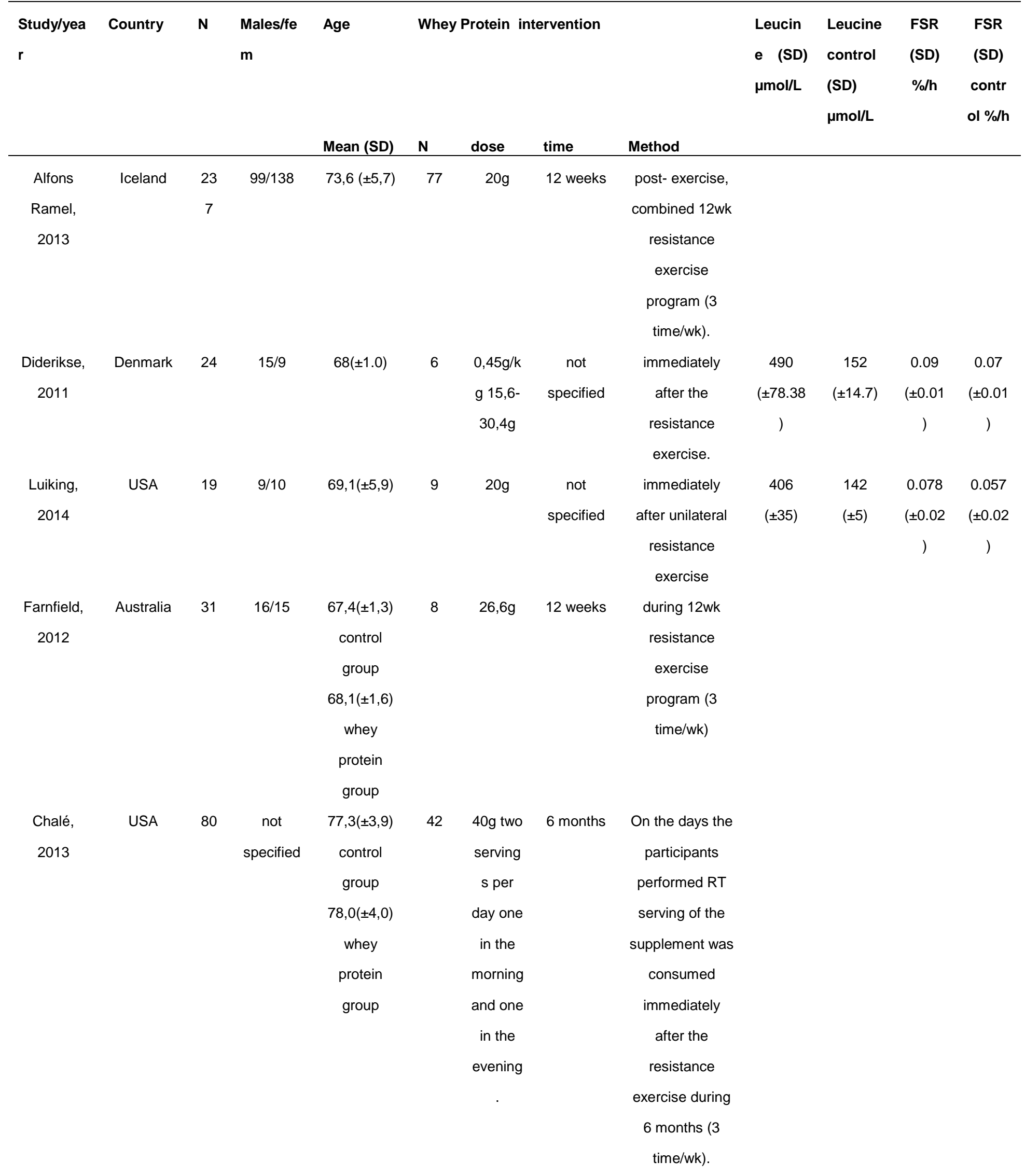


Table 1. Characteristics of included studies

Five primary studies, involving 391 patients met the criteria and were analyzed. The characteristics of all included studies are summarized in Table 1. Studies were published from 2011 to 2014. Sample sizes varied from 19 to 237 with a sum of 142 in the supplement groups. All studies enrolled men and women with mean ages ranging from 67.4 years to 78.0 years. Following the study protocol we tried to assess physical function, body composition, muscle strength and adverse events. However, it was not possible to assess physical function and muscle strength due to the lack of data from the included studies.

\section{Risk of bias}

Random sequence generation was adequate in all five included studies. Allocation concealment was adequate in three studies (Chale, Farnfield, Luiking) and two studies were unclear, as they did not describe the concealment processes (Diderikesen and Ramel). Only one study could blind personnel and assessor, since it used placebo and an independent assessor. Incomplete outcome data was dealt with adequately by all studies; they all reported loss to follow up and adjusted analyses accordingly. Selective reporting was unclear for five studies as the studies did not provide enough information regarding study protocol. All studies were considered free of other bias (Figure 2).

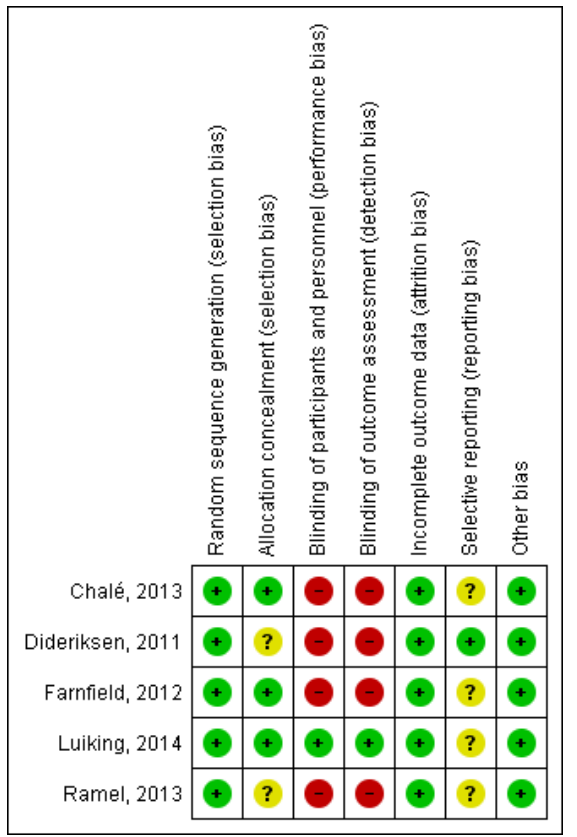

Figure 2. Risk of bias summary: review authors' judgements about each risk of bias item for each included study. 


\section{Primary Outcomes}

\section{Lean Mass and Fat Mass}

Only one study compared lean body mass in the whey protein supplementation group and the control group $(n=80)$, with an average difference of $0.26(95 \% \mathrm{Cl}:-0.43$ to 0.95$)$. The same study compared the fat mass of individuals in the supplementation group and the control group, with an average difference of $-0.12(95 \% \mathrm{Cl} 0.87$ to 0.64$)$ representing a non-significant difference $(p=0.41)$.

\section{Strength}

Two studies assessed strength through 1RM, but due to using different methodologies, it was not possible to compare these studies.

\section{Adverse events}

One study evaluated renal function of participants $(n=144)$ before and after the use of whey protein supplement, by glomerular filtration rate (GFR) and creatinine. The results showed that $20 \mathrm{~g}$ of whey protein supplementation does not negatively affect renal function. [9]

\section{Secondary outcomes}

\section{Total Proteins Ingestion}

Total Proteins Ingestion was analyzed by two trials $(n=203)$, with a total of 107 participants in the experimental group compared with 96 participants in the control group (Ramel et al. and Chale et $a l .$,$) . The results were analyzed used fixed effects model \left(p<0.21, \mathrm{I}^{2}=36\right)$ and standardized mean difference. The supplement whey protein was associated with higher total protein ingestion of SMD $9.40(95 \% \mathrm{Cl} 4.03$ to 14.78$)$ (Figure 3).

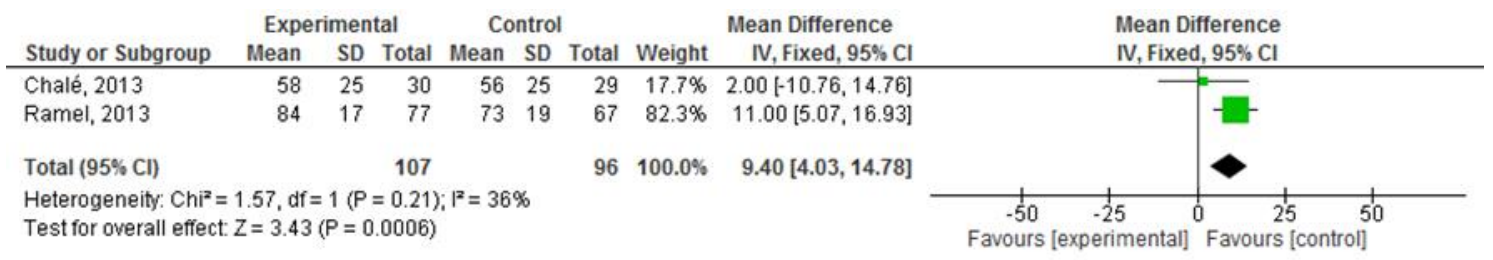

Figure 3. Forest plot of the total proteins ingestion

Leucine concentration

Two studies (Dideriksen and Luiking) analyzed the plasma leucine concentration in control and experimental groups after exercise $(n=31)$. The supplementation of whey protein was associated with a mean difference in plasma leucine concentration ranging from $406 \mu \mathrm{mol} / \mathrm{L}$ to $490 \mu \mathrm{mol} / \mathrm{L}$ compared with the control group (Figure 4). Considering the evidence of heterogeneity $\left(p<0.05, I^{2}=0.74\right)$, we applied the random-effects model.

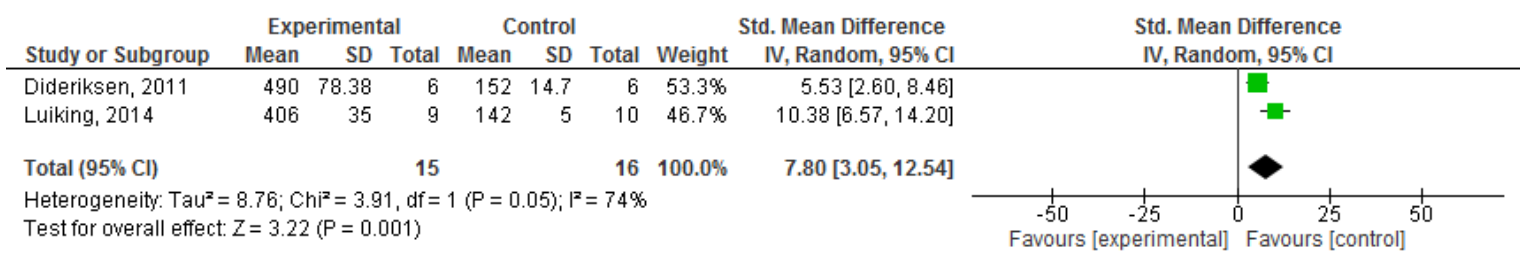

Figure 4. Forest plot of the leucine concentration 
Mixed muscle protein synthesis rate (FSR) was also analyzed by Dideriksen and Luiking. The supplementation was associated with increased FSR. The results were analyzed used fixed effects model $\left(p<0.34, l^{2}=0\right)$ and standardized mean difference. The effect of whey protein supplementation on FSR was SMD 1.26 (95\% Cl 0.46 to 2.07 ) compared with that of the control group (Figure 5).

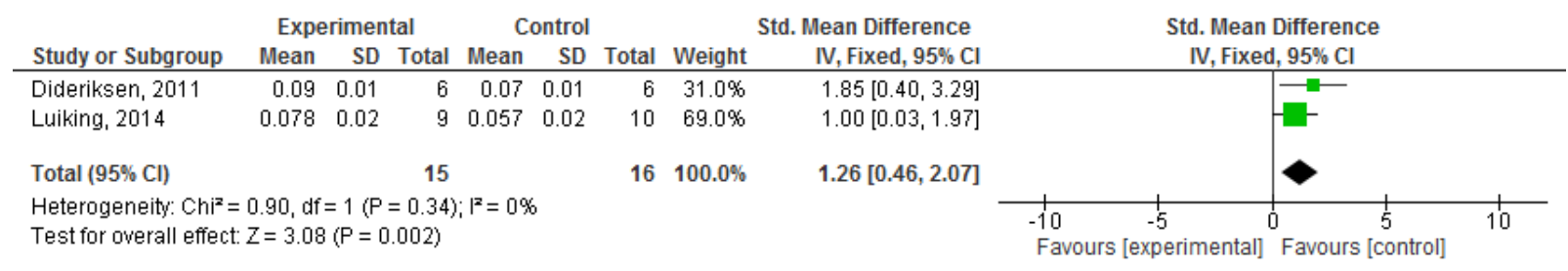

Figure 5. Forest plot of mixed muscle protein synthesis rate

\section{Heterogeneity}

We found substantial heterogeneity between studies for total muscle protein ingestion (chisquare=1.57; $p<0.21 ; I^{2}=36 \%$ ), leucine (chi-square= 8.76; $p<0.05 ; I^{2}=74 \%$ ) and mixed muscle protein synthesis (chi-square $=0.90 ; p<0.34 ; 12=0 \%$ ).

\section{Quality of evidence}

Using the GRADE quality of evidence, outcomes are classified on a scale from high to low across different outcomes. The quality of the evidence is considered low due to lack of blinding in most studies, unclear allocation concealment, and imprecision of results due to wide confidence intervals (and specifically in Chale and Luiking studies). Additionally, inconsistency in the results (forest plots) heterogeneity could have been caused by intensity and type of the intervention (exercise) and different whey protein dosages. The interpretation of a low quality of evidence means that future studies are very likely to change the estimates of whey protein and resistance exercise for the elderly.

\section{Discussion}

Degradation processes and protein synthesis are dynamic processes, which operate to control the gain or loss of muscle mass. Muscle hypertrophy occurs when protein synthesis rate is greater than the rate of degradation; conversely the loss of muscle mass occurs when muscle protein degradation rate is greater than the synthesis [13]. Elderly people have changes in protein metabolism resulting in decreased muscle mass, muscle strength, and physical performance [14]. Additionally, aging is accompanied by reduced consumption of nutrients [15]. The elderly generally has slower chewing, less hunger and thirst, reducing food intake compared to young adults. The food intake of this population reduces approximately $25 \%$ between 40 and 70 years of age [16].

Sarcopenia is defined as loss of function and muscle mass due to aging. It is highly relevant for clinical practice, since it is associated with various negative outcomes, including decreased functionality and survival [17]. Additionally, elderly go through losses in performing daily activities, resulting in increased risk of falls, fractures, disability, dependence, periodic hospitalizations and increased mortality $[2,18]$. The prevalence of sarcopenia in the elderly, among the ages of 60-70 years, varies between $5-13 \%$. In the elderly over 80 years, the prevalence is between $11-50 \%$. Even with a conservative estimate of prevalence, sarcopenia affected around 50 million people in 2014 and will affect an estimated 200 million over the next 40 years. Thus, there is growing interest in the potential of supplements to help fight the consequences of the ageing process. 
One published meta-analysis on the effect of protein timing on muscle strength and hypertrophy included 34 studies analyzing different protein supplements in adults and elderly. The authors reported no significant effects of protein supplementation on strength nor hypertrophy [10]. A more recent systematic review and meta-analysis analyzed nine studies on the effect of protein supplementation in elderly submitted to resistance training. The authors found no association between protein supplementation and changes in muscle mass and muscle strength. In conclusion, the authors recommended further research into each type of protein supplementation individually [11]. Thus our review specifically focused on whey protein supplementation and found five randomized controlled trials which evaluated the effect of whey protein supplementation in elderly submitted to resistance training. Only one of these trials was also included in the Finger et al. systematic review.

The results of the present systematic review indicate that despite the increased concentration of leucine and mixed muscle protein synthesis rate (FSR) after consumption of whey protein supplementation, there was no increase in muscle mass in the experimental group. Additionally, we evaluated the impact of whey protein supplementation on physical function, body composition, muscle strength and adverse events; however, data for these outcomes are scarce. It is worth noting the lack of studies assessing outcomes related to the effect of supplementation on aspects of clinical sarcopenia and its effectiveness on physical function, muscle strength and body composition, which are important outcomes for a patient's quality of life. There are more emphasis in publishing biochemical outcomes than physical fitness outcomes such as strength and body composition, showing a concern with the mechanism of action.

In a review about sarcopenia Dodds and Sayer report that three points are essential in dietary treatment for sarcopenia: protein, vitamin $D$ and antioxidants [17]. In a study on the protein intake requirement comparing older and younger men, the authors identified that the daily intake of protein to increase muscle protein synthesis is greater in the elderly than in adults [19]. The amino acids, especially branched chain amino acids, and leucine in particular, have an important role to stimulate the signaling pathway mTOR [20]. Leucine is one of the most cited amino acids in muscle protein synthesis. Norton and Layman suggest leucine as the key stimulus for muscle protein synthesis by the activation of factors of the mammalian target of rapamycin (mTOR) [21]. In a study with adult men Churchward-Venne showed the positive effect of supplementation of whey protein in muscle protein synthesis, through their quantity of amino acids, particularly leucine, and suggested whey protein as an important supplement for those who do not consume adequate amounts of protein in the meal [22]. One of the assumptions that would indicate the use of whey protein supplementation would be a rapid digestibility compared to other protein supplements [23]. In response to Resistance Training muscle activation and damage occurs in which stimulates protein turnover [3]. If the body amino acid levels are insufficient, the result is a negative protein balance, leading to harmful side effects such as loss of muscle mass and recovery of the late period [4]. A clinical trial by Farnfield et al. with young men demonstrated that the ingestion of the whey protein supplementation immediately after resistance exercise has an effect on increasing the activation of kinases translational compared with exercise alone [24].

The industry of nutrition supplements invest in the development of different types of protein supplements for muscle growth. Despite interest in the potential of whey protein to increase muscle strength in the elderly, the current evidence does not support its widespread consumption. In addition, excessive consumption of proteins is related to development of diseases. A narrative review on the adverse effects of excess protein consumption listed the most likely disorders and health risks in adults, which included disorders of bone and calcium homeostasis, disorders of renal function, increased cancer risk, disorders of liver function, and precipitated progression of coronary artery disease [12]. Thus further research is needed to understand both the benefits and potential harms of whey protein before recommending this substance is recommended. 


\title{
Limitations
}

The main limitation of this systematic review, as well as other systematic reviews on the subject, is the lack of randomized controlled trials in the elderly over 60 years. Some studies mixed elderly and young data, hindering the use of these data in our study. Subgroup analysis considering gender could not be done; therefore it is not known whether the efficacy of whey protein differs by gender. This is plausible given the different body composition of men and women. In addition, a lack of data were found on important outcomes such as physical function and muscle strength.

\section{Conclusions}

In conclusion, our review found that although there was an increase in total protein intake, resulting in increased concentration of leucine and mixed muscle protein synthesis rate, no significant increases in muscle mass or strength were found. Some outcomes considered important in our initial protocol could not be evaluated due to a lack of research related to these outcomes. Further research is needed to justify the supplementation of whey protein in the elderly.

Author Contributions: Provided material support and supervised the research: M.I.R. Prepared the manuscript: T.C and A.J.G. Study selection and Analyzed the data: T.C., A.J.G and M.I.R. Assisted in drafting and revising the manuscript: KM and CF.

Conflicts of Interest: The authors declare no conflict of interest.

\author{
Abbreviations \\ FSR: Mixed muscle protein synthesis rate \\ MD: Mean difference \\ $\mathrm{Cl}$ : Confidence interval \\ SMD: Standardized mean difference \\ MR Maximum repitition
}

\section{References}

1. Csapo, R.; Alegre, L.M. Effects of resistance training with moderate vs heavy loads on muscle mass and strength in the elderly: A meta-analysis. Scand J Med Sci Sports 2015, 24, 12536.

2. Janssen, I.; Baumgartner, R.N.; Ross, R.; Rosenberg, I.H.; Roubenoff, R. Skeletal muscle cutpoints associated with elevated physical disability risk in older men and women. Am J Epidemiol 2004, 159, 413 421.

3. Campbell, B.; Kreider, R.B.; Ziegenfuss, T.; La Bounty, P.; Roberts, M.; Burke, D.; Landis, J.; Lopez, H.; Antonio, J. International society of sports nutrition position stand: Protein and exercise. J Int Soc Sports Nutr. 2007 Sep 26;4:8.

4. Wilborn, C.D.; Taylor, L.W.; Outlaw, J.; Williams, L.; Campbell, B.; Foster, C.A.; Smith-Ryan, A.; Urbina, S.; Hayward, S. The effects of pre- and post-exercise whey vs. Casein protein consumption on body composition and performance measures in collegiate female athletes. J Sports Sci Med 2013, 12, 74-79.

5. Chen, W.C.; Huang, W.C.; Chiu, C.C.; Chang, Y.K.; Huang, C.C. Whey protein improves exercise performance and biochemical profiles in trained mice. Med Sci Sports Exerc 2014, 46, 1517-1524.

6. Liberati, A.; Altman, D.G.; Tetzlaff, J.; Mulrow, C.; Gotzsche, P.C.; loannidis, J.P.; Clarke, M.; Devereaux, P.J.; Kleijnen, J.; Moher, D. The prisma statement for reporting systematic reviews and meta-analyses of studies that evaluate health care interventions: Explanation and elaboration. J Clin Epidemiol 2009, 62, 23.

7. DerSimonian, R.; Laird, N. Meta-analysis in clinical trials. Control Clin Trials 1986, 7, 177-188. 
8. Chale, A.; Cloutier, G.J.; Hau, C.; Phillips, E.M.; Dallal, G.E.; Fielding, R.A. Efficacy of whey protein supplementation on resistance exercise-induced changes in lean mass, muscle strength, and physical function in mobility-limited older adults. J Gerontol A Biol Sci Med Sci 2013, 68, 682-690.

9. Ramel, A.; Arnarson, A.; Geirsdottir, O.G.; Jonsson, P.V.; Thorsdottir, I. Glomerular filtration rate after a $12-$ wk resistance exercise program with post-exercise protein ingestion in community dwelling elderly. Nutrition 2013, 29, 719-723.

10. Schoenfeld, B.J.; Aragon, A.A.; Krieger, J.W. The effect of protein timing on muscle strength and hypertrophy: A meta-analysis. J Int Soc Sports Nutr 2013, 10, 1550-2783.

11. Finger, D.; Goltz, F.R.; Umpierre, D.; Meyer, E.; Rosa, L.H.; Schneider, C.D. Effects of protein supplementation in older adults undergoing resistance training: A systematic review and meta-analysis. Sports Med 2015, 45, 245-255.

12. Delimaris, I. Adverse effects associated with protein intake above the recommended dietary allowance for adults. ISRN Nutr 2013, 18.

13. Gordon, B.S.; Kelleher, A.R.; Kimball, S.R. Regulation of muscle protein synthesis and the effects of catabolic states. Int J Biochem Cell Biol 2013, 45, 2147-2157.

14. Dodds, R.M.; Roberts, H.C.; Cooper, C.; Sayer, A.A. The epidemiology of sarcopenia. J Clin Densitom 2015, 12, 00051-00057.

15. Budui, S.L.; Rossi, A.P.; Zamboni, M. The pathogenetic bases of sarcopenia. Clin Cases Miner Bone Metab 2015, 12, 22-26.

16. Nieuwenhuizen, W.F.; Weenen, H.; Rigby, P.; Hetherington, M.M. Older adults and patients in need of nutritional support: Review of current treatment options and factors influencing nutritional intake. Clin Nutr 2010, 29, 160-169.

17. Dodds, R.; Sayer, A.A. Sarcopenia. Arq Bras Endocrinol Metabol 2014, 58, 464-469.

18. Masanes, F.; Culla, A.; Navarro-Gonzalez, M.; Navarro-Lopez, M.; Sacanella, E.; Torres, B.; Lopez-Soto, A. Prevalence of sarcopenia in healthy community-dwelling elderly in an urban area of barcelona (spain). J Nutr Health Aging 2012, 16, 184-187.

19. Moore, D.R.; Churchward-Venne, T.A.; Witard, O.; Breen, L.; Burd, N.A.; Tipton, K.D.; Phillips, S.M. Protein ingestion to stimulate myofibrillar protein synthesis requires greater relative protein intakes in healthy older versus younger men. J Gerontol A Biol Sci Med Sci 2015, 70, 57-62.

20. Sancak, Y.; Bar-Peled, L.; Zoncu, R.; Markhard, A.L.; Nada, S.; Sabatini, D.M. Ragulator-rag complex targets mtorc1 to the lysosomal surface and is necessary for its activation by amino acids. Cell 2010, 141, 290-303.

21. Norton, L.E.; Layman, D.K. Leucine regulates translation initiation of protein synthesis in skeletal muscle after exercise. J Nutr 2006, 136, 533S-537S.

22. Churchward-Venne, T.A.; Burd, N.A.; Mitchell, C.J.; West, D.W.; Philp, A.; Marcotte, G.R.; Baker, S.K.; Baar, K.; Phillips, S.M. Supplementation of a suboptimal protein dose with leucine or essential amino acids: Effects on myofibrillar protein synthesis at rest and following resistance exercise in men. J Physiol 2012, 590, 2751-2765.

23. Devries, M.C.; Phillips, S.M. Supplemental protein in support of muscle mass and health: Advantage whey. J Food Sci 2015, 80, A8-A15.

24. Farnfield, M.M.; Carey, K.A.; Gran, P.; Trenerry, M.K.; Cameron-Smith, D. Whey protein ingestion activates mtor-dependent signalling after resistance exercise in young men: A double-blinded randomized controlled trial. Nutrients 2009, 1, 263-275. 
25. Luiking, Y.C.; Deutz, N.E.; Memelink, R.G.; Verlaan, S.; Wolfe, R.R. Postprandial muscle protein synthesis is higher after a high whey protein, leucine-enriched supplement than after a dairy-like product in healthy older people: A randomized controlled trial. Nutr J 2014, 13, 1475-2891.

26. Dideriksen, K.J.; Reitelseder, S.; Petersen, S.G.; Hjort, M.; Helmark, I.C.; Kjaer, M.; Holm, L. Stimulation of muscle protein synthesis by whey and caseinate ingestion after resistance exercise in elderly individuals. Scand J Med Sci Sports 2011, 21, 1600-0838. 